\title{
Nonlinear integral inequalities with delay for discontinuous functions and their applications
}

\author{
Yuzhen Mi, Shengfu Deng ${ }^{*}$ and Xiaopei Li
}

"Correspondence: sfdeng@vt.edu; sf_deng@sohu.com Department of Mathematics, Zhanjiang Normal University, Zhanjiang, Guangdong 524048, China

\begin{abstract}
This paper investigates integral inequalities with delay for discontinuous functions involving two nonlinear terms. We do not require the classes $\wp$ and $J$ in Gallo and Piccirillo's paper (Bound. Value Probl. 2009:808124, 2009). Our main results can be applied to generalize Gallo and Piccirillo's results and lovane's results (Nonlinear Anal., Theory Methods Appl. 66:498-508, 2007). Examples to show the bounds of solutions of an impulsive differential equation are also given, which can not be estimated by Gallo and Piccirillo's results.
\end{abstract}

MSC: 26D15; 26D20

Keywords: integral inequalities; discontinuous functions; nonlinear; impulsive differential equations

\section{Introduction}

The Gronwall-Bellman integral inequalities and their various linear and nonlinear generalizations, involving continuous or discontinuous functions, play very important roles in investigating different qualitative characteristics of solutions for differential equations and impulsive differential equations such as existence, uniqueness, continuation, boundedness, continuous dependence of parameters, stability, attraction, practical stability. The literature on inequalities for continuous functions and their applications is vast (see [1-8]). Recently, more attention has been paid to generalizations of Gronwall-Bellman's results for discontinuous functions (see [9-17]) and their applications (see [11, 18, 19]). Among them, one of the important things is that Samoilenko and Perestyuk [17] studied the following inequality

$$
u(x) \leq c+\int_{x_{0}}^{x} f(s) u(s) d s+\sum_{x_{0}<x_{i}<x} \beta_{i} u\left(x_{i}-0\right)
$$

about the nonnegative piecewise continuous function $u(x)$, where $c, \beta_{i}$ are nonnegative constants, $f(s)$ is a positive function, and $x_{i}$ are the first kind discontinuity points of the function $u(x)$. Then Borysenko [20] considered

$$
\begin{aligned}
& u(x) \leq c+\int_{x_{0}}^{x} f(s) u^{m}(s) d s+\sum_{x_{0}<x_{i}<x} \beta_{i} u\left(x_{i}-0\right), \quad m>0, m \neq 1, \\
& u(x) \leq c+\int_{x_{0}}^{x} f(s) u^{m}(s) d s+\sum_{x_{0}<x_{i}<x} \beta_{i} u^{m}\left(x_{i}-0\right), \quad m>0, m \neq 1 .
\end{aligned}
$$

\section{Springer}

O $2013 \mathrm{Mi}$ et al.; licensee Springer. This is an Open Access article distributed under the terms of the Creative Commons Attribution License (http://creativecommons.org/licenses/by/2.0), which permits unrestricted use, distribution, and reproduction in any medium, provided the original work is properly cited. 
He replaced the constant $c$ by a positive monotonously nondecreasing function $a(x)$, and also estimated the inequalities

$$
\begin{aligned}
& u(x) \leq a(x)+\int_{x_{0}}^{x} f(s) u(s) d s+\sum_{x_{0}<x_{i}<x} \beta_{i} u^{m}\left(x_{i}-0\right), \quad m>0, \\
& u(x) \leq a(x)+\int_{x_{0}}^{x} f(s) u^{m}(s) d s+\sum_{x_{0}<x_{i}<x} \beta_{i} u^{m}\left(x_{i}-0\right), \quad m>0, m \neq 1 .
\end{aligned}
$$

In 2005 , he [18] generalized the inequalities above from one integral to two integrals with a form

$$
\begin{aligned}
u(x) \leq & c+\int_{x_{0}}^{x} q(s) u(s) d s+\int_{x_{0}}^{x} q(s) \int_{x_{0}}^{s} g(\tau) u^{m}(\tau) d \tau d s \\
& +\sum_{x_{0}<x_{i}<x} \beta_{i} u^{m}\left(x_{i}-0\right), \quad m>0 .
\end{aligned}
$$

In 2007, Iovane [21] investigated the inequalities with delay

$$
\begin{aligned}
& u(x) \leq a(x)+\int_{x_{0}}^{x} f(s) u(b(s)) d s+\sum_{x_{0}<x_{i}<x} \beta_{i} u^{m}\left(x_{i}-0\right), \quad m>0, \\
& u(x) \leq a(x)+\int_{x_{0}}^{x} f(s) u^{m}(b(s)) d s+\sum_{x_{0}<x_{i}<x} \beta_{i} u^{m}\left(x_{i}-0\right), \quad m>0, m \neq 1 .
\end{aligned}
$$

Later, Gallo and Piccirillo [22] further discussed

$$
u(x) \leq a(x)+h(x) \int_{x_{0}}^{x} f(s) w(u(b(s))) d s+\sum_{x_{0}<x_{i}<x} \beta_{i} u^{m}\left(x_{i}-0\right)
$$

with a general nonlinear term $w(u)$ of $u$. They assumed that $w \in \wp$ or $w \in J$, where the class $\wp$ consists of all nonnegative, nondecreasing and continuous functions $w(u)$ on $[0, \infty)$ such that $w(0)=0$ and $w(\alpha u) \leq w(\alpha) w(u)$ for all $\alpha>0$ and $u \geq 0$, and the class $J$ consists of all positive, nondecreasing and continuous functions $w(u)$ on $(0, \infty)$ such that $w(0)=0$ and $w\left(\alpha^{-1} u\right)>\alpha^{-1} w(u)$ for all $\alpha \geq 1$ and $u>0$. The classes $\wp$ and $J$ allow a reduction of $a(t)$ to the case of a constant $a_{0}$ by dividing $a(x)$ if $a(x)$ is a positive and nondecreasing function. Actually, when we study behaviors of solutions of impulsive differential equations, $a(x)$ may not be a nondecreasing function, and $w$ may not satisfy the condition $w \in \wp$ or $w \in J$. For example, $w(u)=e^{u}$ does not belong to the class $\wp$ and $J$ for any $\alpha>1$ and large $u>0$. Thus, it is interesting to avoid such conditions.

Motivated by this observation, in this paper, we consider the following much more general inequality

$$
\begin{aligned}
u(x) \leq & a(x)+\int_{b_{1}\left(x_{0}\right)}^{b_{1}(x)} f_{1}(x, s) w_{1}(u(s)) d s+\int_{b_{2}\left(x_{0}\right)}^{b_{2}(x)} f_{2}(x, s) w_{2}(u(s)) d s \\
& +\sum_{x_{0}<x_{i}<x} \beta_{i} u^{m}\left(x_{i}-0\right), \quad m>0
\end{aligned}
$$

with two nonlinear terms $w_{1}(u)$ and $w_{2}(u)$ of $u$, where we do not restrict $w_{1}$ and $w_{2}$ to the class $\wp$ or the class $J$. We also show that many integral inequalities for discontinuous 
functions such as (1.3), (1.4) and (1.6) can be reduced to the form of (1.7). Our main result is applied to estimate the bounds of solutions of an impulsive ordinary differential equation.

\section{Main results}

Consider (1.7), and assume that

$\left(\mathrm{C}_{1}\right) w_{1}(x)$ and $w_{2}(x)$ are continuous and nondecreasing functions on $[0, \infty)$ and are positive on $(0, \infty)$ such that $\frac{w_{2}(x)}{w_{1}(x)}$ is nondecreasing;

$\left(C_{2}\right) a(x)$ is defined on $\left[x_{0}, \infty\right)$ and $a\left(x_{0}\right) \neq 0 ; \beta_{i}$ is a nonnegative constant for any positive integer $i$;

(C $\left.C_{3}\right) f_{1}(x, s)$ and $f_{2}(x, s)$ are continuous and nonnegative functions on $\left[x_{0}, \infty\right) \times\left[x_{0}, \infty\right)$;

$\left(\mathrm{C}_{4}\right) b_{1}(x)$ and $b_{2}(x)$ are continuously differentiable and nondecreasing such that $x_{0} \leq$ $b_{1}(x) \leq x$ and $x_{0} \leq b_{2}(x) \leq x$ on $\left[x_{0}, \infty\right)$;

$\left(\mathrm{C}_{5}\right)$ For $x \in\left[x_{0}, \infty\right), u(x)$ is nonnegative and piecewise-continuous with the first kind of discontinuities at the points $x_{i}: x_{0}<x_{1}<\cdots$, where $i$ is a nonnegative integer and $\lim _{i \rightarrow \infty} x_{i}=\infty$.

Let $W_{j}(u)=\int_{\tilde{u}_{j}}^{u} \frac{d z}{w_{j}(z)}$ for $u \geq \tilde{u}_{j}$ and $j=1,2$, where $\tilde{u}_{j}$ is a given positive constant. Clearly, $W_{j}$ is strictly increasing so its inverse $W_{j}^{-1}$ is well defined, continuous and increasing in its corresponding domain.

Theorem 2.1 Suppose that $\left(C_{k}\right)(k=1, \ldots, 5)$ hold, and $u(x)$ satisfies (1.7) for a positive constant $m$. Let $u_{i}(x)=u(x)$ for $x \in\left[x_{i}, x_{i+1}\right)$. Then the estimate of $u(x)$ is recursively given by for $x \in\left[x_{i}, x_{i+1}\right), i=0,1,2, \ldots$,

$$
u_{i}(x) \leq W_{2}^{-1}\left[W_{2} \circ W_{1}^{-1}\left(W_{1}\left(r_{i+1}(x)\right)+\int_{b_{1}\left(x_{i}\right)}^{b_{1}(x)} \tilde{f}_{1}(x, s) d s\right)+\int_{b_{2}\left(x_{i}\right)}^{b_{2}(x)} \tilde{f}_{2}(x, s) d s\right],
$$

where

$$
\begin{aligned}
\tilde{f}_{j}(x, s)= & \max _{x_{0} \leq \tau \leq x} f_{j}(\tau, s), \quad j=1,2, \quad r_{1}(x)=\max _{x_{0} \leq \tau \leq x}|a(\tau)|, \\
r_{i+1}(x)= & r_{1}(x)+\sum_{k=1}^{i} \int_{b_{1}\left(x_{k-1}\right)}^{b_{1}\left(x_{k}\right)} \tilde{f}_{1}(x, s) w_{1}\left(u_{k-1}(s)\right) d s \\
& +\sum_{k=1}^{i} \int_{b_{2}\left(x_{k-1}\right)}^{b_{2}\left(x_{k}\right)} \tilde{f}_{2}(x, s) w_{2}\left(u_{k-1}(s)\right) d s+\sum_{k=1}^{i} \beta_{k} u_{k-1}^{m}\left(x_{k}-0\right),
\end{aligned}
$$

provided that

$$
\begin{aligned}
& W_{1}\left(r_{i+1}(x)\right)+\int_{b_{1}\left(x_{i}\right)}^{b_{1}(x)} \tilde{f}_{1}(x, s) d s \leq \int_{\tilde{u}_{1}}^{\infty} \frac{d z}{w_{1}(z)} \\
& W_{2} \circ W_{1}^{-1}\left(W_{1}\left(r_{i+1}(x)\right)+\int_{b_{1}\left(x_{i}\right)}^{b_{1}(x)} \tilde{f}_{1}(x, s) d s\right)+\int_{b_{2}\left(x_{i}\right)}^{b_{2}(x)} \tilde{f}_{2}(x, s) d s \leq \int_{\tilde{u}_{2}}^{\infty} \frac{d z}{w_{2}(z)} .
\end{aligned}
$$

The proof is given in Section 3.

Remark 2.1 (1) If $w_{j}$ satisfies $\int_{\tilde{u}_{j}}^{\infty} \frac{d z}{w_{j}(z)}=\infty$ for $j=1,2$, then $i$ in Theorem 2.1 can be any nonzero integer. [6] pointed out that different choices of $\tilde{u}_{j}$ in $W_{j}$ do not affect our results for $j=1,2$. If $a(x) \equiv 0$, then define $W_{1}(0)=0$, and (2.1) is still true. 
(2) Take $b_{1}(x)=x, a(x)=c, f_{1}(t, s)=f(s), f_{2}(t, s)=0, w_{1}(u)=u$ and $m=1$. Hence, (1.7) becomes (1.1). It is easy to check that $W_{1}(u)=\ln \frac{u}{\tilde{u}_{1}}$ and $W_{1}^{-1}(u)=\tilde{u}_{1} e^{u}$. From Theorem 2.1, we know that for $x \in\left[x_{i}, x_{i+1}\right)$,

$$
u_{i}(x) \leq r_{i+1}(x) e^{\int_{x_{i}}^{x} f(s) d s}
$$

with

$$
r_{i+1}(x)=c+\sum_{k=1}^{i} \int_{x_{k-1}}^{x_{k}} f(s) u_{k-1}(s) d s+\sum_{k=1}^{i} \beta_{k} u_{k-1}\left(x_{k}-0\right) .
$$

Hence,

$$
\begin{aligned}
r_{1}(x) & =c, \quad u_{0}(x) \leq c e^{\int_{x_{0}}^{x} f(s) d s}, \\
r_{2}(x) & =c+\int_{x_{0}}^{x_{1}} f(s) u_{0}(s) d s+\beta_{1} u_{0}\left(x_{1}-0\right) \\
& \leq c+\int_{x_{0}}^{x_{1}} f(s) c e^{\int_{x_{0}}^{s} f(\tau) d \tau} d s+c \beta_{1} e^{\int_{x_{0}}^{x_{1}} f(s) d s} \\
& =c+\left.c e^{\int_{x_{0}}^{s} f(\tau) d \tau}\right|_{x_{0}} ^{x_{1}}+c \beta_{1} e^{\int_{x_{0}}^{x_{1}} f(s) d s}=c\left(1+\beta_{1}\right) e^{\int_{x_{0}}^{x_{1}} f(s) d s}, \\
u_{1}(x) & \leq c\left(1+\beta_{1}\right) e^{\int_{x_{0}}^{x} f(s) d s} .
\end{aligned}
$$

After recursive calculations, we have for $x \geq x_{0}$

$$
u(x) \leq c \Pi_{x_{0}<x_{k}<x}\left(1+\beta_{k}\right) e^{\int_{x_{0}}^{x} f(s) d s},
$$

which is same as the one in [17].

(3) Clearly, (1.2) and (1.3) are special cases of (1.7). If $b^{\prime}(x)>0$ on $\left[x_{0}, \infty\right)$, then (1.6) can be rewritten as

$$
u(x) \leq a(x)+h(x) \int_{b\left(x_{0}\right)}^{b(x)} \frac{f\left(b^{-1}(s)\right)}{b^{\prime}\left(b^{-1}(s)\right)} w(u(s)) d s+\sum_{x_{0}<x_{i}<x} \beta_{i} u^{m}\left(x_{i}-0\right) .
$$

Let $f_{1}(x, s)=h(x) \frac{f\left(b^{-1}(s)\right)}{b^{\prime}\left(b^{-1}(s)\right)}$ and $f_{2}(x, s) \equiv 0$, the inequality above is same as (1.7). Similarly, (1.5) can also be reduced to (1.7).

Consider the inequality

$$
\begin{aligned}
u(x) \leq & a(x)+\int_{x_{0}}^{x} g_{1}(x, s) \int_{x_{0}}^{s} h_{1}(s, \tau) w_{1}(u(\tau)) d \tau d s \\
& +\int_{x_{0}}^{x} g_{2}(x, s) \int_{x_{0}}^{s} h_{2}(s, \tau) w_{2}(u(\tau)) d \tau d s+\sum_{x_{0}<x_{i}<x} \beta_{i} u^{m}\left(x_{i}-0\right)
\end{aligned}
$$

which looks more complicated than (1.7). 
Corollary 2.1 Suppose that $\left(\mathrm{C}_{1}\right)-\left(\mathrm{C}_{3}\right)$ and $\left(\mathrm{C}_{5}\right)$ hold, and that the functions $g_{j}$ and $h_{j}(j=$ $1,2)$ are both nonnegative and continuous on $\left[x_{0}, \infty\right) \times\left[x_{0}, \infty\right)$. If $(2.4)$ holds, then for $x \in$ $\left[x_{i}, x_{i+1}\right), i=0,1,2, \ldots$,

$$
\begin{aligned}
u_{i}(x) \leq & W_{2}^{-1}\left[W_{2} \circ W_{1}^{-1}\left(W_{1}\left(r_{i+1}(x)\right)+\int_{x_{i}}^{x} \int_{s}^{x} \max _{x_{0} \leq \tau \leq x} g_{1}(\tau, v) h_{1}(v, s) d v d s\right)\right. \\
& \left.+\int_{x_{i}}^{x} \int_{s}^{x} \max _{x_{0} \leq \tau \leq x} g_{2}(\tau, v) h_{2}(v, s) d v d s\right],
\end{aligned}
$$

where $r_{i+1}$ and its related functions are defined as in Theorem 2.1 by replacing $f_{j}(x, s)$ with $\int_{s}^{x} \max _{x_{0} \leq \tau \leq x} g_{j}(\tau, v) h_{j}(v, s) d v, j=1,2$.

Proof Because $f_{j}, h_{j}$ and $w_{j}$ are continuous, we have

$$
\begin{aligned}
& \int_{x_{0}}^{x} g_{j}(x, s) \int_{x_{0}}^{s} h_{j}(s, \tau) w_{j}(u(\tau)) d \tau d s \\
& \quad=\int_{x_{0}}^{x} w_{j}(u(\tau)) \int_{\tau}^{x} g_{j}(x, s) h_{j}(s, \tau) d s d \tau \\
& =\int_{x_{0}}^{x} w_{j}(u(s)) \int_{s}^{x} g_{j}(x, \tau) h_{j}(\tau, s) d \tau d s \leq \int_{x_{0}}^{x} f_{j}(x, s) w_{j}(u(s)) d s,
\end{aligned}
$$

where $f_{j}(x, s):=\int_{s}^{x} \max _{x_{0} \leq \tau \leq x} g_{j}(\tau, v) h_{j}(v, s) d v$. Then $(2.4)$ is reduced to

$$
\begin{aligned}
u(x) \leq & a(x)+\int_{x_{0}}^{x} f_{1}(x, s) w_{1}(u(s)) d s+\int_{x_{0}}^{x} f_{2}(x, s) w_{2}(u(s)) d s \\
& +\sum_{x_{0}<x_{i}<x} \beta_{i} u^{m}\left(x_{i}-0\right),
\end{aligned}
$$

which is just the form of (1.7), if we take $b_{j}(x)=x$ for $j=1,2$. Note that for fixed $s$, the function $f_{j}(x, s)$ is increasing in $x$. So $\tilde{f}_{j}(x, s):=\max _{t_{0} \leq \tau \leq x} f_{j}(\tau, s)=f_{j}(x, s)$. By Theorem 2.1, for $x \in\left[x_{i}, x_{i+1}\right), i=0,1,2, \ldots$,

$$
\begin{aligned}
u_{i}(x) \leq & W_{2}^{-1}\left[W_{2} \circ W_{1}^{-1}\left(W_{1}\left(r_{i+1}(x)\right)+\int_{x_{i}}^{x} \int_{s}^{x} \max _{x_{0} \leq \tau \leq x} g_{1}(\tau, v) h_{1}(v, s) d v d s\right)\right. \\
& \left.+\int_{x_{i}}^{x} \int_{s}^{x} \max _{x_{0} \leq \tau \leq x} g_{2}(\tau, v) h_{2}(v, s) d v d s\right] .
\end{aligned}
$$

Remark 2.2 Using the same way, we can change inequality (1.4) into the form of (1.7) with $a(x)=c, f_{1}(x, s)=q(s), f_{2}(x, s)=g(s) \int_{s}^{x} q(\tau) d \tau, w_{1}(u)=u$ and $w_{2}(u)=u^{m}$.

\section{Proof of Theorem 2.1}

Obviously, $r_{1}(x)$ is positive and nondecreasing in $x$, and $\tilde{f}_{j}(x, s)$ is nonnegative and nondecreasing in $x$ for each fixed $s$ and $j=1,2$. They satisfy $r_{1}(x) \geq a(x)$ and $\tilde{f}_{j}(x, s) \geq f_{j}(x, s)$.

We first consider $x \in\left[x_{0}, x_{1}\right)$, and we have from (1.7) and (2.2)

$$
\begin{aligned}
u(x) & \leq a(x)+\int_{b_{1}\left(x_{0}\right)}^{b_{1}(x)} f_{1}(x, s) w_{1}(u(s)) d s+\int_{b_{2}\left(x_{0}\right)}^{b_{2}(x)} f_{2}(x, s) w_{2}(u(s)) d s \\
& \leq r_{1}(x)+\int_{b_{1}\left(x_{0}\right)}^{b_{1}(x)} f_{1}(x, s) w_{1}(u(s)) d s+\int_{b_{2}\left(x_{0}\right)}^{b_{2}(x)} f_{2}(x, s) w_{2}(u(s)) d s
\end{aligned}
$$


Take any fixed $T \in\left(x_{0}, x_{1}\right)$, and we investigate the following inequality

$$
u(x) \leq r_{1}(T)+\int_{b_{1}\left(x_{0}\right)}^{b_{1}(x)} \tilde{f}_{1}(T, s) w_{1}(u(s)) d s+\int_{b_{2}\left(x_{0}\right)}^{b_{2}(x)} \tilde{f}_{2}(T, s) w_{2}(u(s)) d s
$$

for $x \in\left[x_{0}, T\right]$, where $\tilde{f}_{1}$ and $\tilde{f}_{2}$ are defined in (2.2). Let

$$
z(x)=\int_{b_{1}\left(x_{0}\right)}^{b_{1}(x)} \tilde{f}_{1}(T, s) w_{1}(u(s)) d s+\int_{b_{2}\left(x_{0}\right)}^{b_{2}(x)} \tilde{f}_{2}(T, s) w_{2}(u(s)) d s
$$

and $z\left(x_{0}\right)=0$. Hence, $u(x) \leq r_{1}(T)+z(x)$. Clearly, $z(x)$ is a nonnegative, nondecreasing and differentiable function for $x \in\left(x_{0}, T\right]$. Moreover, $b_{j}(x)$ is differentiable and nondecreasing in $x \in\left[x_{0}, T\right]$ for $j=1,2$. Thus, $b_{j}^{\prime}(x) \geq 0$ for $x \in\left[x_{0}, T\right]$. Since $w_{1}$ and $w_{2}$ are nondecreasing, $z(x)+r_{1}(T)>0$ and $b_{j}(x) \leq x$ for $x \in\left[x_{0}, T\right]$, we have

$$
\begin{aligned}
\frac{z^{\prime}(x)}{w_{1}\left(z(x)+r_{1}(T)\right)} \leq & \frac{b_{1}^{\prime}(x) \tilde{f}_{1}\left(T, b_{1}(x)\right) w_{1}\left(u\left(b_{1}(x)\right)\right)}{w_{1}\left(z(x)+r_{1}(T)\right)}+\frac{b_{2}^{\prime}(x) \tilde{f}_{2}\left(T, b_{2}(x)\right) w_{2}\left(u\left(b_{2}(x)\right)\right)}{w_{1}\left(z(x)+r_{1}(T)\right)} \\
\leq & \frac{b_{1}^{\prime}(x) \tilde{f}_{1}\left(T, b_{1}(x)\right) w_{1}\left(z\left(b_{1}(x)\right)+r_{1}(T)\right)}{w_{1}\left(z(x)+r_{1}(T)\right)} \\
& +\frac{b_{2}^{\prime}(x) \tilde{f}_{2}\left(T, b_{2}(x)\right) w_{2}\left(z\left(b_{2}(x)\right)+r_{1}(T)\right)}{w_{1}\left(z(x)+r_{1}(T)\right)} \\
\leq & \frac{b_{1}^{\prime}(x) \tilde{f}_{1}\left(T, b_{1}(x)\right) w_{1}\left(z(x)+r_{1}(T)\right)}{w_{1}\left(z(x)+r_{1}(T)\right)} \\
& +\frac{b_{2}^{\prime}(x) \tilde{f}_{2}\left(T, b_{2}(x)\right) w_{2}\left(z\left(b_{2}(x)\right)+r_{1}(T)\right)}{w_{1}\left(z(x)+r_{1}(T)\right)} \\
\leq & b_{1}^{\prime}(x) \tilde{f}_{1}\left(T, b_{1}(x)\right)+\frac{b_{2}^{\prime}(x) \tilde{f}_{2}\left(T, b_{2}(x)\right) w_{2}\left(z\left(b_{2}(x)\right)+r_{1}(T)\right)}{w_{1}\left(z\left(b_{2}(x)\right)+r_{1}(T)\right)} .
\end{aligned}
$$

Integrating both sides of the inequality above, from $x_{0}$ to $x$, we obtain

$$
\begin{aligned}
W_{1}\left(z(x)+r_{1}(T)\right) \leq & W_{1}\left(r_{1}(T)\right)+\int_{x_{0}}^{x} b_{1}^{\prime}(s) \tilde{f}_{1}\left(T, b_{1}(s)\right) d s \\
& +\int_{x_{0}}^{x} b_{2}^{\prime}(s) \tilde{f}_{2}\left(T, b_{2}(s)\right) \phi\left(z\left(b_{2}(s)\right)+r_{1}(T)\right) d s \\
\leq & W_{1}\left(r_{1}(T)\right)+\int_{b_{1}\left(x_{0}\right)}^{b_{1}(x)} \tilde{f}_{1}(T, s) d s+\int_{b_{2}\left(x_{0}\right)}^{b_{2}(x)} \tilde{f}_{2}(T, s) \phi\left(z(s)+r_{1}(T)\right) d s
\end{aligned}
$$

for $x_{0}<x \leq T$, where $\phi(x)=\frac{w_{2}(x)}{w_{1}(x)}$, or equivalently,

$$
\xi(x) \leq W_{1}\left(r_{1}(T)\right)+\int_{b_{1}\left(x_{0}\right)}^{b_{1}(x)} \tilde{f}_{1}(T, s) d s+\int_{b_{2}\left(x_{0}\right)}^{b_{2}(x)} \tilde{f}_{2}(T, s) \phi\left(W_{1}^{-1}(\xi(s))\right) d s \triangleq z_{1}(x),
$$

where

$$
\xi(x)=W_{1}\left(z(x)+r_{1}(T)\right) .
$$


It is easy to check that $\xi(x) \leq z_{1}(x), z_{1}\left(x_{0}\right)=W_{1}\left(r_{1}(T)\right)$ and $z_{1}(x)$ is differentiable, positive and nondecreasing on $\left(x_{0}, T\right]$. Since $\phi\left(W_{1}^{-1}(u)\right)$ is nondecreasing from the assumption $\left(\mathrm{C}_{1}\right)$, we have by $(2.3)$

$$
\begin{aligned}
& \frac{z_{1}^{\prime}(x)}{\phi\left(W_{1}^{-1}\left(z_{1}(x)\right)\right)} \\
& \quad \leq \frac{\tilde{f}_{1}\left(T, b_{1}(x)\right) b_{1}^{\prime}(x)}{\phi\left(W_{1}^{-1}\left(z_{1}(x)\right)\right)}+\frac{\tilde{f}_{2}\left(T, b_{2}(x)\right) \phi\left(W_{1}^{-1}\left(\xi\left(b_{2}(x)\right)\right)\right) b_{2}^{\prime}(x)}{\phi\left(W_{1}^{-1}\left(z_{1}(x)\right)\right)} \\
& \quad \leq \frac{\tilde{f}_{1}\left(T, b_{1}(x)\right) b_{1}^{\prime}(x)}{\phi\left(W_{1}^{-1}\left(z_{1}(x)\right)\right)}+\frac{\tilde{f}_{2}\left(T, b_{2}(x)\right) \phi\left(W_{1}^{-1}\left(z_{1}\left(b_{2}(x)\right)\right)\right) b_{2}^{\prime}(x)}{\phi\left(W_{1}^{-1}\left(z_{1}(x)\right)\right)} \\
& \quad \leq \frac{\tilde{f}_{1}\left(T, b_{1}(x)\right) b_{1}^{\prime}(x)}{\phi\left(W_{1}^{-1}\left(W_{1}\left(r_{1}(T)\right)+\int_{b_{1}\left(x_{0}\right)}^{b_{1}(x)} \tilde{f}_{1}(T, s) d s\right)\right)}+\tilde{f}_{2}\left(T, b_{2}(x)\right) b_{2}^{\prime}(x) .
\end{aligned}
$$

Note that

$$
\begin{aligned}
\int_{x_{0}}^{x} \frac{z_{1}^{\prime}(s)}{\phi\left(W_{1}^{-1}\left(z_{1}(s)\right)\right)} d s & =\int_{x_{0}}^{x} \frac{w_{1}\left(W_{1}^{-1}\left(z_{1}(s)\right)\right) z_{1}^{\prime}(s)}{w_{2}\left(W_{1}^{-1}\left(z_{1}(s)\right)\right)} d s=\int_{W_{1}^{-1}\left(z_{1}\left(x_{0}\right)\right)}^{W_{1}^{-1}\left(z_{1}(x)\right)} \frac{d u}{w_{2}(u)} \\
& =W_{2} \circ W_{1}^{-1}\left(z_{1}(x)\right)-W_{2} \circ W_{1}^{-1}\left(z_{1}\left(x_{0}\right)\right) \\
& =W_{2} \circ W_{1}^{-1}\left(z_{1}(x)\right)-W_{2}\left(r_{1}(T)\right) .
\end{aligned}
$$

Integrating both sides of inequality (3.3), from $x_{0}$ to $x$, we obtain

$$
\begin{aligned}
W_{2} & \circ W_{1}^{-1}\left(z_{1}(x)\right)-W_{2}\left(r_{1}(T)\right) \\
& =\int_{x_{0}}^{x} \frac{z_{1}^{\prime}(s)}{\phi\left(W_{1}^{-1}\left(z_{1}(s)\right)\right)} d s \\
& \leq \int_{x_{0}}^{x} \frac{\tilde{f}_{1}\left(T, b_{1}(s)\right) b_{1}^{\prime}(s)}{\phi\left(W_{1}^{-1}\left(W_{1}\left(r_{1}(T)\right)+\int_{b_{1}\left(x_{0}\right)}^{b_{1}(s)} \tilde{f}_{1}(T, \tau) d \tau\right)\right)} d s+\int_{x_{0}}^{x} \tilde{f}_{2}\left(T, b_{2}(s)\right) b_{2}^{\prime}(s) d s \\
& \leq W_{2} \circ W_{1}^{-1}\left(W_{1}\left(r_{1}(T)\right)+\int_{b_{1}\left(x_{0}\right)}^{b_{1}(x)} \tilde{f}_{1}(T, s) d s\right)-W_{2}\left(r_{1}(T)\right)+\int_{b_{2}\left(x_{0}\right)}^{b_{2}(x)} \tilde{f}_{2}(T, s) d s .
\end{aligned}
$$

Thus,

$$
W_{2} \circ W_{1}^{-1}\left(z_{1}(x)\right) \leq W_{2} \circ W_{1}^{-1}\left(W_{1}\left(r_{1}(T)\right)+\int_{b_{1}\left(x_{0}\right)}^{b_{1}(x)} \tilde{f}_{1}(T, s) d s\right)+\int_{b_{2}\left(x_{0}\right)}^{b_{2}(x)} \tilde{f}_{2}(T, s) d s .
$$

We have by (2.3)

$$
\begin{aligned}
u(x) & \leq z(x)+r_{1}(T) \leq W_{1}^{-1}(\xi(x)) \leq W_{1}^{-1}\left(z_{1}(x)\right) \\
& \leq W_{2}^{-1}\left[W_{2} \circ W_{1}^{-1}\left(W_{1}\left(r_{1}(T)\right)+\int_{b_{1}\left(x_{0}\right)}^{b_{1}(x)} \tilde{f}_{1}(T, s) d s\right)+\int_{b_{2}\left(x_{0}\right)}^{b_{2}(x)} \tilde{f}_{2}(T, s) d s\right] .
\end{aligned}
$$

Since the inequality above is true for any $x \in\left[x_{0}, T\right]$, we obtain

$$
u(T) \leq W_{2}^{-1}\left[W_{2} \circ W_{1}^{-1}\left(W_{1}\left(r_{1}(T)\right)+\int_{b_{1}\left(x_{0}\right)}^{b_{1}(T)} \tilde{f}_{1}(T, s) d s\right)+\int_{b_{2}\left(x_{0}\right)}^{b_{2}(T)} \tilde{f}_{2}(T, s) d s\right] .
$$


Replacing $T$ by $x$ yields

$$
u(x) \leq W_{2}^{-1}\left[W_{2} \circ W_{1}^{-1}\left(W_{1}\left(r_{1}(x)\right)+\int_{b_{1}\left(x_{0}\right)}^{b_{1}(x)} \tilde{f}_{1}(x, s) d s\right)+\int_{b_{2}\left(x_{0}\right)}^{b_{2}(x)} \tilde{f}_{2}(x, s) d s\right]
$$

This means that (2.1) is true for $x \in\left[x_{0}, x_{1}\right)$ and $i=0$ if replace $u(x)$ with $u_{0}(x)$.

For $i=1$ and $x \in\left[x_{1}, x_{2}\right)$, (1.7) becomes

$$
\begin{aligned}
u(x) \leq & r_{1}(x)+\int_{b_{1}\left(x_{0}\right)}^{b_{1}\left(x_{1}\right)} f_{1}(x, s) w_{1}\left(u_{0}(s)\right) d s+\int_{b_{2}\left(x_{0}\right)}^{b_{2}\left(x_{1}\right)} f_{2}(x, s) w_{2}\left(u_{0}(s)\right) d s \\
& +\beta_{1} u_{0}^{m}\left(x_{1}-0\right)+\int_{b_{1}\left(x_{1}\right)}^{b_{1}(x)} f_{1}(x, s) w_{1}(u(s)) d s+\int_{b_{2}\left(x_{1}\right)}^{b_{2}(x)} f_{2}(x, s) w_{2}(u(s)) d s \\
\leq & r_{2}(x)+\int_{b_{1}\left(x_{1}\right)}^{b_{1}(x)} f_{1}(x, s) w_{1}(u(s)) d s+\int_{b_{2}\left(x_{1}\right)}^{b_{2}(x)} f_{2}(x, s) w_{2}(u(s)) d s,
\end{aligned}
$$

where the definition of $r_{2}(x)$ is given in (2.2). Note that the estimate of $u_{0}(x)$ is known. Equation (3.5) is same as (3.1) if replace $r_{1}(x)$ and $x_{0}$ by $r_{2}(x)$ and $x_{1}$. Thus, by (3.4), we have

$$
u(x) \leq W_{2}^{-1}\left[W_{2} \circ W_{1}^{-1}\left(W_{1}\left(r_{2}(x)\right)+\int_{b_{1}\left(x_{1}\right)}^{b_{1}(x)} \tilde{f}_{1}(x, s) d s\right)+\int_{b_{2}\left(x_{1}\right)}^{b_{2}(x)} \tilde{f}_{2}(x, s) d s\right]
$$

This implies that (2.1) is true for $x \in\left[x_{1}, x_{2}\right)$ and $i=1$ if replace $u(x)$ by $u_{1}(x)$.

Assume that (2.1) is true for $x \in\left[x_{i}, x_{i+1}\right)$, i.e.,

$$
u_{i}(x) \leq W_{2}^{-1}\left[W_{2} \circ W_{1}^{-1}\left(W_{1}\left(r_{i+1}(x)\right)+\int_{b_{1}\left(x_{i}\right)}^{b_{1}(x)} \tilde{f}_{1}(x, s) d s\right)+\int_{b_{2}\left(x_{i}\right)}^{b_{2}(x)} \tilde{f}_{2}(x, s) d s\right]
$$

for $x \in\left[x_{i}, x_{i+1}\right)$.

For $x \in\left[x_{i+1}, x_{i+2}\right)$, (1.7) becomes

$$
\begin{aligned}
u(x) \leq & a(x)+\int_{b_{1}\left(x_{0}\right)}^{b_{1}(x)} f_{1}(x, s) w_{1}(u(s)) d s+\int_{b_{2}\left(x_{0}\right)}^{b_{2}(x)} f_{2}(x, s) w_{2}(u(s)) d s \\
& +\sum_{x_{0}<x_{i+1}<x} \beta_{i+1} u^{m}\left(x_{i+1}-0\right) \\
\leq & r_{1}(x)+\sum_{k=0}^{i} \int_{b_{1}\left(x_{k}\right)}^{b_{1}\left(x_{k+1}\right)} f_{1}(x, s) w_{1}\left(u_{k}(s)\right) d s+\sum_{k=0}^{i} \int_{b_{2}\left(x_{k}\right)}^{b_{2}\left(x_{k+1}\right)} f_{2}(x, s) w_{2}\left(u_{k}(s)\right) d s \\
& +\sum_{k=0}^{i} \beta_{k+1} u_{k}^{m}\left(x_{k+1}-0\right)+\int_{b_{1}\left(x_{i+1}\right)}^{b_{1}(x)} f_{1}(x, s) w_{1}(u(s)) d s \\
& +\int_{b_{2}\left(x_{i+1}\right)}^{b_{2}(x)} f_{2}(x, s) w_{2}(u(s)) d s \\
\leq & r_{i+2}(x)+\int_{b_{1}\left(x_{i+1}\right)}^{b_{1}(x)} f_{1}(x, s) w_{1}(u(s)) d s+\int_{b_{2}\left(x_{i+1}\right)}^{b_{2}(x)} f_{2}(x, s) w_{2}(u(s)) d s
\end{aligned}
$$

where we use the fact that the estimate of $u(x)$ is already known for $x \in\left[x_{0}, x_{i+1}\right)$ by the assumption (3.7). Again (3.8) is same as (3.1) if replace $r_{1}(x)$ and $x_{0}$ by $r_{i+2}(x)$ and $x_{i+1}$. 
Thus, by (3.4), we have

$$
u(x) \leq W_{2}^{-1}\left[W_{2} \circ W_{1}^{-1}\left(W_{1}\left(r_{i+2}(x)\right)+\int_{b_{1}\left(x_{i+1}\right)}^{b_{1}(x)} \tilde{f}_{1}(x, s) d s\right)+\int_{b_{2}\left(x_{i+1}\right)}^{b_{2}(x)} \tilde{f}_{2}(x, s) d s\right]
$$

This yields that (2.1) is true for $x \in\left[x_{i+1}, x_{i+2}\right)$ if replace $u(x)$ by $u_{i+1}(x)$. By induction, we know that (2.1) holds for $x \in\left[x_{i}, x_{i+1}\right)$ for any nonnegative integer $i$. This completes the proof of Theorem 2.1.

\section{Applications}

Consider the following impulsive differential equation

$$
\begin{aligned}
& \frac{d y}{d x}=F(x, y), \quad x \neq x_{i}, \\
& \left.\Delta y\right|_{x=x_{i}}=I_{i}(y),
\end{aligned}
$$

where $y \in \mathbf{R}^{n}, F: \mathbf{R}^{n+1} \rightarrow \mathbf{R}^{n}, I_{i}: \mathbf{R}^{n} \rightarrow \mathbf{R}^{n}(i=1,2, \ldots), x \geq x_{0} \geq 0, \lim _{i \rightarrow \infty} x_{i}=\infty, x_{i-1}<x_{i}$ for all $i=1,2, \ldots$.

Assume that

(1) $\|F(x, y)\| \leq h_{1}(x)\|y\|+h_{2}(x) e^{\|y\|}$, where $h_{1}, h_{2}$ are nonnegative and continuous on $\left[x_{0}, \infty\right)$;

(2) $\left\|I_{i}(y)\right\| \leq \beta_{i}\|y\|^{m}$, where $\beta_{i}$ and $m$ are nonnegative constants.

The solution of (4.1) with an initial value $y\left(x_{0}\right)=y_{0}$ is given by

$$
y(x)=y_{0}+\int_{x_{0}}^{x} F(s, y) d s+\sum_{x_{0}<x_{i}<x} I_{i}\left(y\left(x_{i}-0\right)\right)
$$

which implies that

$$
\|y(x)\| \leq\left\|y_{0}\right\|+\int_{x_{0}}^{x}\left(h_{1}(s)\|y\|+h_{2}(s) e^{\|y\|}\right) d s+\sum_{x_{0}<x_{i}<x} \beta_{i}\left\|y\left(x_{i}-0\right)\right\|^{m} .
$$

Let

$$
\begin{aligned}
& u(x)=\|y(x)\|, \quad a(x) \equiv\left\|y_{0}\right\|, \quad b_{1}(x)=x, \quad b_{2}(x)=x, \\
& f_{1}(x, s)=h_{1}(s), \quad f_{2}(x, s)=h_{2}(s), \quad w_{1}(u)=u, \quad w_{2}(u)=e^{u},
\end{aligned}
$$

so (4.3) is same as (1.7). It is easy to obtain for any positive constants $\tilde{u}_{1}$ and $\tilde{u}_{2}$

$$
\begin{aligned}
& r_{1}(x) \equiv\left\|y_{0}\right\|, \quad \tilde{f}_{1}(x, s)=h_{1}(s), \quad \tilde{f}_{2}(x, s)=h_{2}(s), \quad W_{1}(u)=\int_{\tilde{u}_{1}}^{u} \frac{d z}{w_{1}(z)}=\ln \frac{u}{\tilde{u}_{1}}, \\
& W_{1}^{-1}(u)=\tilde{u}_{1} e^{u}, \quad W_{2}(u)=\int_{\tilde{u}_{2}}^{u} \frac{d z}{w_{2}(u)}=e^{-\tilde{u}_{2}}-e^{-u}, \quad W_{2}^{-1}(u)=-\ln \left(e^{-\tilde{u}_{2}}-u\right), \\
& r_{i+1}(x)=\left\|y_{0}\right\|+\sum_{k=1}^{i} \int_{x_{k-1}}^{x_{k}} h_{1}(s) u_{k-1}(s) d s+\sum_{k=1}^{i} \int_{x_{k-1}}^{x_{k}} h_{2}(s) e^{u_{k-1}(s)} d s \\
& +\sum_{k=1}^{i} \beta_{k} u_{k-1}^{m}\left(x_{k}-0\right) .
\end{aligned}
$$


Thus, for any nonnegative integer $i$ and $x \in\left(x_{i}, x_{i+1}\right)$

$$
u_{i}(x) \leq-\ln \left(e^{-r_{i+1}(x) e^{\int_{x_{i}}^{x} h_{1}(s) d s}}-\int_{x_{i}}^{x} h_{2}(s) d s\right),
$$

provided that

$$
e^{-r_{i+1}(x) e^{\int_{x_{i}}^{x} h_{1}(s) d s}}-\int_{x_{i}}^{x} h_{2}(s) d s>0 .
$$

Remark 4.1 From (4.3), we know that $w_{2}(u)=e^{u}$. Clearly, $w_{2}(2 u)=e^{2 u} \leq w_{2}(2) w_{2}(u)=$ $e^{2} e^{u}$ does not hold for large $u>0$. Thus, $w_{2}(u)$ does not belong to the class $\wp$. Again $w_{2}\left(\frac{u}{2}\right)=$ $e^{\frac{u}{2}} \geq \frac{1}{2} w_{2}(u)=\frac{1}{2} e^{u}$ does not hold for large $u>0$, so $w_{2}(u)$ does not belong to the class $J$. Hence, the results in [22] can not be applied to inequality (4.3).

\section{Competing interests}

The authors declare that they have no competing interests.

\section{Authors' contributions}

All the authors read and approved the final manuscript.

\section{Acknowledgements}

This work was supported by the Project of Department of Education of Guangdong Province, China (No. 2012KJCX0074), the PhD Start-up Fund of the Natural Science Foundation of Guangdong Province, China (No. S2011040000464), the China Postdoctoral Science Foundation-Special Project (No. 201104077), the Scientific Research Foundation for the Returned Overseas Chinese Scholars, State Education Ministry (No. (2012)940), the Natural Fund of Zhanjiang Normal University (No. LZL1101), and the Doctoral Project of Zhanjiang Normal University (No. ZL1109).

\section{Received: 16 May 2013 Accepted: 26 August 2013 Published: 11 September 2013}

\section{References}

1. Agarwal, RP: On an integral inequality in $n$ independent variables. J. Math. Anal. Appl. 85, 192-196 (1982)

2. Agarwal, RP, Deng, S, Zhang, W: Generalization of a retarded Gronwall-like inequality and its applications. Appl. Math. Comput. 165, 599-612 (2005)

3. Bihari, I: A generalization of a lemma of Bellman and its application to uniqueness problems of differential equations. Acta Math. Hung. 7, 81-94 (1956)

4. Borysenko, S, Matarazzo, G, Pecoraro, M: A generalization of Bihari's lemma for discontinuous functions and its application to the stability problem of differential equations with impulse disturbance. Georgian Math. J. 13, 229-238 (2006)

5. Cheung, W: Some new nonlinear inequalities and applications to boundary value problems. Nonlinear Anal. 64 2112-2128 (2006)

6. Choi, SK, Deng, S, Koo, NJ, Zhang, W: Nonlinear integral inequalities of Bihari-type without class H. Math. Inequal. Appl. 8, 643-654 (2005)

7. Pachpatte, BG: Integral inequalities of the Bihari type. Math. Inequal. Appl. 5, 649-657 (2002)

8. Pinto, M: Integral inequalities of Bihari-type and applications. Funkc. Ekvacioj 33, 387-403 (1990)

9. Banov, D, Simeonov, P: Integral Inequalities and Applications. Mathematics and Its Applications, vol. 57. Kluwer Academic, Dordrecht (1992)

10. Borysenko, S, lovane, G: About some new integral inequalities of Wendroff type for discontinuous functions. Nonlinear Anal. 66, 2190-2203 (2007)

11. Borysenko, SD, Toscano, S: Impulsive differential systems: The problem of stability and practical stability. Nonlinear Anal. 71, e1843-e1849 (2009)

12. Hu, SC, Lakshmikantham, V, Leela, S: Impulsive differential systems and the pulse phenomena. J. Math. Anal. Appl. $137,605-612(1989)$

13. Iovane, G: On Gronwall-Bellman-Bihari type integral inequalities in several variables with retardation for discontinuous functions. Math. Inequal. Appl. 11, 599-606 (2008)

14. Lakshmikantham, V, Bainov, DD, Simeonov, PS: Theory of Impulsive Differential Equations. Series in Modern Applied Mathematics, vol. 6. World Scientific, Teaneck (1989)

15. Mitropolskiy, YuA, Samoilenko, AM, Perestyuk, N: On the problem of substantiation of overoging method for the second equations with impulse effect. Ukr. Mat. Zh. 29, 750-762 (1977)

16. Mitropolskiy, YuA, lovane, G, Borysenko, SD: About a generalization of Bellman-Bihari type inequalities for discontinuous functions and their applications. Nonlinear Anal. 66, 2140-2165 (2007)

17. Samoilenko, AM, Perestyuk, N: Differential Equations with Impulse Effect. Visha Shkola, Kyiv (1987)

18. Borysenko, SD, Ciarletta, M, lovane, G: Integro-sum inequalities and motion stability of systems with impulse perturbations. Nonlinear Anal. 62, 417-428 (2005) 
19. Borysenko, SD, lovane, G, Giordano, P: Investigations of the properties motion for essential nonlinear systems perturbed by impulses on some hypersurfaces. Nonlinear Anal. 62, 345-363 (2005)

20. Borysenko, SD: About one integral inequality for piece-wise continuous functions, p. 323. In: Proc. Int. Kravchuk Conf. Kyiv (2004)

21. Iovane, G: Some new integral inequalities of Bellman-Bihari type with delay for discontinuous functions. Nonlinear Anal. 66, 498-508 (2007)

22. Gallo, A, Piccirillo, AM: On some generalizations Bellman-Bihari result for integro-functional inequalities for discontinuous functions and their applications. Bound. Value Probl. 2009, Article ID 808124 (2009)

doi:10.1186/1029-242X-2013-430

Cite this article as: Mi et al.: Nonlinear integral inequalities with delay for discontinuous functions and their applications. Journal of Inequalities and Applications 2013 2013:430.

Submit your manuscript to a SpringerOpen ${ }^{\circ}$ journal and benefit from:

- Convenient online submission

- Rigorous peer review

- Immediate publication on acceptance

- Open access: articles freely available online

- High visibility within the field

- Retaining the copyright to your article

Submit your next manuscript at $>$ springeropen.com 